\title{
NOTAS ACERCA DE LA DISTRIBUCIÓN DE PAPILIONIDAE (LEPIDOPTERA: PAPILIONOIDEA) EN EL NORTE DE LA ORINOQUIA COLOMBIANA*
}

\author{
Juan Carlos Agudelo M. ${ }^{1}$, Néstor Pérez-Buitrago ${ }^{2}$
}

\begin{abstract}
Resumen
Se revisaron 56 ejemplares de mariposas de la familia Papilionidae colectados esporádicamente en el norte de la Orinoquia colombiana en municipios pertenecientes a los departamentos de Arauca, Norte de Santander y Casanare, en un rango altitudinal entre los 120 y 494 msnm. Los especímenes corresponden a tres géneros, siete especies y siete subespecies siendo las más abundantes Battus polydamas polydamas y Parides eurimedes arriphus con 15 y 12 individuos, respectivamente. Las siete especies identificadas corresponden al $11,5 \%$ de las 61 reportadas para Colombia y al 29,2\% de las 24 especies de Papilionidae registradas para toda la región Orinoquia. A nivel regional la riqueza de Papilioniade en Arauca es igual a la reportada en una localidad en el Meta (siete sp.) y menor que la del departamento de Casanare (nueve sp.). Reportamos la ampliación de la distribución geográfica de cuatro subespecies a nivel regional.
\end{abstract}

Palabras clave: biodiversidad, Orinoquia, colecciones biológicas, distribución, Papilionidae, Lepidoptera

\section{NOTES ON THE DISTRIBUTION OF PAPILIONIDAE (LEPIDOPTERA: PAPILIONOIDEA) IN THE NORTHERN COLOMBIAN ORINOCO}

\begin{abstract}
Fifty six specimens of Papilionidae butterflies were non-systematically collected and are documented from the northern part of the Colombian Orinoquia. Specifically, specimens were collected in Arauca, Casanare, and Norte de Santander departments in an altitudinal gradient between 120 and 494 m.a.s.l. The specimens belong to three genera, seven species and seven subspecies being the most abundant Battus polydamas polydamas and Parides eurimedes arriphus with fifteen and twelve individuals respectively. The seven species identified correspond to $11.5 \%$ of the 61species reported to Colombia and 29.2 $\%$ of the 24 species of this family registered for the whole Colombian Orinoco region. At regional scale, the species richness of Papilionidae in Arauca equals $t$ reported in a locality of Meta department (seven sp.) and lesser than the department of Casanare (nine sp.). For four subspecies, the collection localities imply the enlargement of their previously reported geographical distribution, at regional spatial scale.
\end{abstract}

Key words: biodiversity, Orinoquia, biological collection, distribution, Papillionidae, Lepidoptera.

\footnotetext{
*FR: 26-II-2014 . FA: 4-II-2015

${ }^{1}$ Estudiante M.Sc Ciencias Agrarias con énfasis en Entomología. Curador Colección Entomológica de la Orinoquia (CEO), Grupo de Investigación en Ciencias de la Orinoquia (GICO), Universidad Nacional de Colombia sede Orinoquia. Arauca, Colombia. Autor para correspondencia. E-mail: jcagudelo@gmail.com

${ }^{2}$ Ph.D. Biología. Profesor Asistente Universidad Nacional de Colombia sede Orinoquia, Grupo de Investigación en Ciencias de la Orinoquia (GICO). Arauca, Colombia. E-mail: nfperezb@unal.edu.co 


\section{INTRODUCCIÓN}

La región biogeográfica de la Orinoquia abarca los departamentos de Guainía, Guaviare, Arauca, Casanare, Meta y Vichada (MEDINA et al., 2010) representando el 20,2\% de la superficie nacional. La biodiversidad de la Orinoquia es una de las menos documentadas de Colombia y a nivel regional tiene pocas áreas protegidas. Recientemente, se han propuesto 17 áreas prioritarias para la conservación y uso sostenible de la biodiversidad, de las cuales el corredor Meta-Casanare, el Lipa, el corredor Cocuy-río Casanare-valle aluvial Meta, y los humedales de Arauca se encuentran total o parcialmente en la jurisdicción del departamento de Arauca (QUINTERO et al., 2007; ROSALES et al., 2010).

Los ecosistemas de la Orinoquia se encuentran seriamente amenazados por actividades humanas como la deforestación intensiva para la implementación de monocultivos de palma de aceite (Elaeis guineensis) y arroz (Oryza sativa), la ganadería, la minería y la extracción de hidrocarburos con proyectos como el de Caño Limón que opera desde 1986 (PEÑA-PIRAZAN, 2003; FERNÁNDEZ et al., 2010), y más recientemente el proyecto Chipirón que se está implementando en el estero el Lipa. Estas dinámicas de desarrollo, que responden a políticas estatales, marcan un precedente potencialmente negativo para una región donde el estado del conocimiento de los procesos ecológicos y los patrones de diversidad son mínimos. Por ejemplo, recientemente en un estudio bibliométrico de ARBELÁEZ-CORTÉS (2013) relacionado con publicaciones científicas de diversidad biológica para Colombia, se reportó que el departamento de Arauca presenta valores mínimos para todos los parámetros evaluados (i.e. número de estudios de biodiversidad, listas de taxones, nuevos registros, artículos de conservación, estudios de botánica, estudios de entomología, y estudios de vertebrados) respecto a los demás departamentos colombianos.

Algunas causas para justificar esta situación de inactividad investigativa en Arauca y otros departamentos de la Orinoquia, están relacionadas con problemas de seguridad asociados al conflicto armado (ARBELÁEZ-CORTÉS, 2013), las precarias vías de acceso a potenciales sitios de estudio y la ausencia de instituciones que de forma consistente incluyan la Orinoquia en sus agendas de investigación. Esta situación demanda generar esfuerzos para cuantificar la diversidad biológica idealmente en todos sus componentes (riqueza y abundancia de especies, diversidad genética y diversidad ecosistémica).

En ambientes como la Orinoquia, donde la información disponible es escasa, una estrategia viable consiste en fortalecer las colecciones biológicas existentes en la región, o como en el caso de Arauca crear nuevas colecciones biológicas. La importancia de estas iniciativas es que permiten sentar las bases de conocimiento para establecer una perspectiva histórica de la biodiversidad y sus dinámicas espacio-temporales (PONDER et al., 2001), y por lo tanto son indispensables para comprender tanto las reales dimensiones de la diversidad como la verdadera magnitud de su pérdida (PONDER et al., 2001; GROPP, 2003; SUARÉZ \& TSUTSUI, 2004). Con este objetivo en mente, en 2012 se crea la Colección Entomológica de la Orinoquia (CEO) de la Universidad Nacional de Colombia sede Orinoquia, la cual cuenta con más de 3300 especímenes de los cuales 1536 corresponden al orden Lepidoptera. Este orden es el segundo orden más abundante de insectos con alrededor de 200.000 especies en el mundo (LLORENTE \& MARTÍNEZ, 1998). 
Para Colombia, el número estimado de especies de mariposas es de 3019, de las cuales 61 pertenecen a la familia Papilionidae, lo cual corresponde al $47 \%$ de las especies de este taxon listadas para el Neotrópico, y casi el 12\% de los papiliónidos del mundo (ANDRADE, 2002; LAMAS, 2004). Estos valores posicionan a Colombia como uno de los países más diversos junto con Brasil y México (LLORENTE \& MARTÍNEZ, 1998).

En este estudio reportamos un listado de especies registradas de la familia Papilionidae colectadas en diferentes municipios de la región norte de la Orinoquia colombiana, que no han sido previamente documentadas en la literatura y que, por lo tanto, constituyen un aporte para el conocimiento detallado de la distribución de las especies reportadas.

\section{MATERIALES Y MÉTODOS}

Todos los especímenes colectados se encuentran depositados en la Colección Entomológica de la Orinoquia (CEO) de la sede Orinoquia de la Universidad Nacional de Colombia y provienen en su mayoría del departamento de Arauca, y cuatro registros adicionales del piedemonte de los departamentos de Casanare y Norte de Santander. Los especímenes fueron colectados mediante red entomológica, sacrificados por presión torácica, y almacenados en sobres de papel milano. Para cada espécimen se registró la localidad detallada (incluidas coordenadas geográficas), altitud, fecha, hora, colector, hábitat y comentarios. Posteriormente fueron trasladados a las instalaciones de la Universidad Nacional sede Orinoquia, en donde se realizó el proceso de montaje siguiendo la metodología propuesta por VILLARREAL et al. (2006). La identificación se llevó a cabo por comparación con diferentes fuentes bibliográficas (ROTHSCHILD \& JORDAN, 1906; APOLINAR, 1939, 1940; GARCÍA-ROBLEDO et al., 2002; LECROM et al., 2002; LAMAS, 2004) y la corroboración de algunos especímenes por parte del especialista Gonzalo Andrade del Instituto de Ciencias Naturales de la Universidad Nacional de Colombia.

El ecosistema predominante en la zona de estudio es el de sabana inundable, el cual presenta una temperatura media anual de $30^{\circ} \mathrm{C}$, y una humedad relativa $80 \%$ promedio en época lluviosa y de 65\% en temporada seca. El área de estudio presenta paisajes geomorfológicos de llanura con preponderancia de sabanas y escasas apariciones de bosques de galería y matas de monte. La precipitación media anual cercana a $2200 \mathrm{~mm}$, con un régimen monomodal, que presenta un periodo de lluvias de ocho meses comprendidos desde abril hasta noviembre, en el cual se registra el 95\% de la lluvia del año, y un periodo seco de cuatro meses iniciando en diciembre y finalizando en marzo (RIPPSTEIN et al., 2001).

Las colectas fueron realizadas entre el 2 de julio de 2010 y el 14 de diciembre de 2013 mediante muestreos no sistemáticos y esporádicos en ambientes de bosque de galería, bosque secundario y sabana inundable en los municipios de Arauca, Arauquita, Saravena, Tame, Fortul, Paz de Ariporo y Toledo (Figura 1), en un rango altitudinal entre los 120 a 494 msnm. 


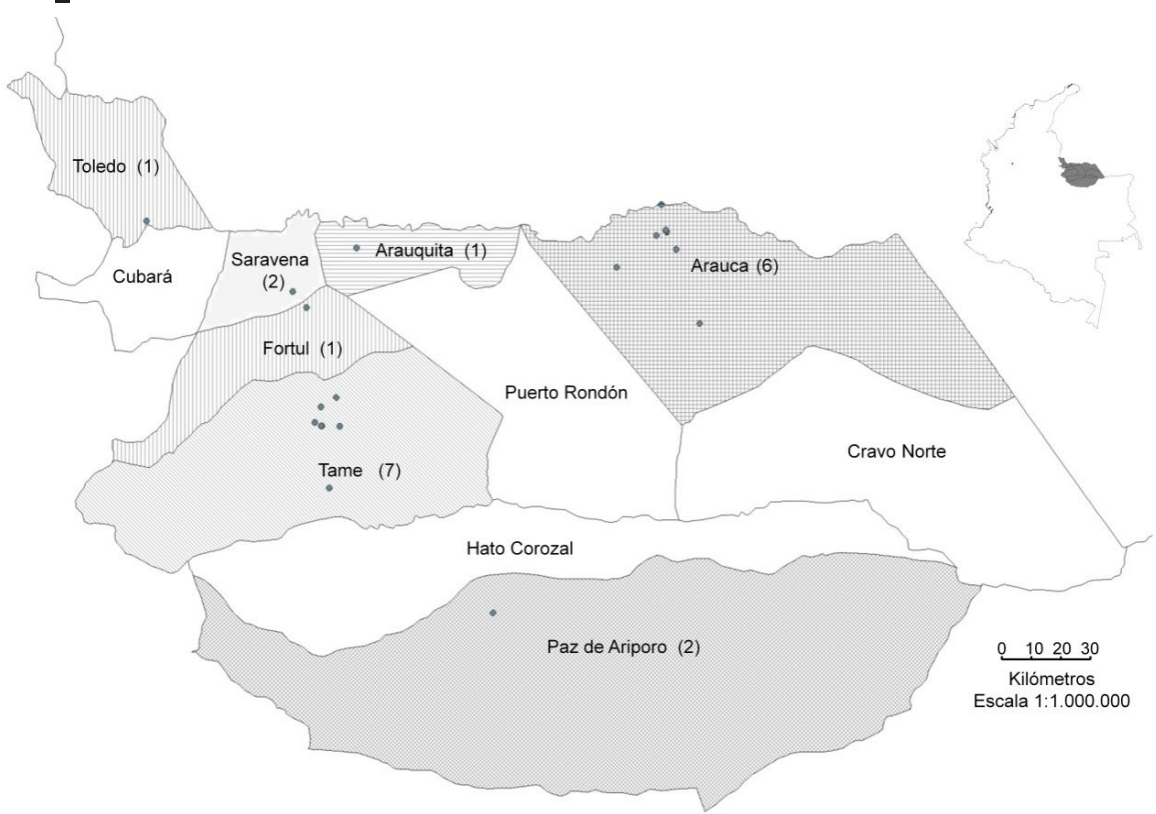

Figura 1. Municipios con representación de Papilionidae en la Colección Entomológica de la Orinoquia (CEO) de la Universidad Nacional de Colombia. Entre paréntesis número de especies y subespecies por municipio.

\section{RESULTADOS}

Se revisaron 56 ejemplares correspondientes a tres géneros, siete especies y siete subespecies. La subespecie mejor representada es Battus polydamas polydamas (Linnaeus, 1758) con 15 especímenes, seguida de las subespecies Parides eurimedes arriphus (Boisduval, 1836) con 12 especímenes y P. eurimedes mycale (Godman \& Salvin, 1890) con 10. La subespecie Heraclides thoas nealces (Rothschild \& Jordan, 1906) y la especie H. homothoas (Rothschild \& Jordan, 1906) se encuentran representadas en la colección con seis ejemplares, mientras que $H$. anchisiades idaeus (Fabricius, 1793) reporta tres individuos. La subespecie P. sesostris tarquinus (Boisduval, 1836) reporta dos registros en la colección y P. eurimedes eurimedes (Stoll, 1782) junto con B. ingenuus (Dyar, 1907) se encuentran representadas en la colección por un espécimen cada una. Arauca es el municipio mejor representado en la colección con 30 especímenes seguido de Tame con 17 individuos, mientras que los restantes municipios están representados por un número entre uno y tres especímenes (Tabla 1). La mayor riqueza de especies la registra el municipio de Tame con siete especies y subespecies registradas, seguido de Arauca con seis, Saravena junto con Paz de Ariporo registran dos especies, mientras que Fortul, Arauquita y Toledo tienen un registro cada uno (Figura 1). 
Tabla 1. Número de especímenes por especie o subespecie colectados en cada uno de los municipios del norte de la región de la Orinoquia colombiana.

\begin{tabular}{|c|c|c|c|c|c|c|c|}
\hline $\begin{array}{ll}\text { Especie } & \text { Municipio } \\
\end{array}$ & Arauca & Arauquita & Fortul & Tame & Toledo & $\begin{array}{l}\text { Paz de } \\
\text { Ariporo }\end{array}$ & Saravena \\
\hline Battus polydamas polydamas & 12 & & & 1 & & 2 & \\
\hline Parides eurimedes arriphus & 5 & & & 6 & & & 1 \\
\hline Heraclides thoas nealces & 1 & & 1 & 3 & 1 & & \\
\hline Heracllides homothoas & 5 & & & & & 1 & \\
\hline Parides eurimedes mycale & 4 & & & 4 & & & 2 \\
\hline Parides sesostris tarquinius & & 1 & & 1 & & & \\
\hline Heraclides anchisiades idaeus & 3 & & & & & & \\
\hline Parides eurimedes eurimedes & & & & 1 & & & \\
\hline Battus ingenuus & & & & 1 & & & \\
\hline
\end{tabular}

\section{DISCUSIÓN}

Este trabajo constituye el primer reporte de la lepidopterofauna de la familia Papilionidae para el departamento de Arauca, en donde se destacan los registros obtenidos en los municipios de Arauca, Arauquita, Saravena y Tame, principalmente. Las siete especies identificadas y reportadas corresponden al 11,5\% de las 61 especies de Papilionidae reportadas para Colombia y al 29,2\% de las 24 especies registradas para la región Orinoquia (ANDRADE, 2002). Es notable que, a pesar de lo esporádico de las colectas realizadas en este estudio, la riqueza de Papilionidae en Arauca es igual a la de una localidad del departamento del Meta con siete especies (FRAIJA \& FAJARDO, 2006) y levemente inferior que el departamento de Casanare (nueve especies) (YARA-ORTIZ et al., 2011).

La distribución geográfica de las especies en la región Orinoquia revela que las especies: $H$. thoas, $P$. eurimedes y $H$. anchisiades son comunes a los departamentos de Arauca, Casanare y Meta; H. homothoas y B. polydamas son comunes a los departamentos de Casanare y Arauca, mientras que $P$. sesostris tarquinius y Battus ingennus son raras para Arauca (Tabla 2).

Adicionalmente, se destaca la ampliación de la distribución geográfica de las especies $P$. eurimedes arriphus, $H$. thoas nealces, $H$. homothoas y $P$. eurimedes mycale. Para el caso de P. eurimides arriphus, de acuerdo a ANDRADE (2002), su distribución geográfica correspondía a la región del Chocó biogeográfico hasta el flanco oriental de la Cordillera Central; mientras que LECROM et al. (2002) indican que se trata de una especie endémica de la región biogeográfica del MagdalenaNechí, que corresponde a zonas en los departamentos de Cundinamarca, Tolima, Huila, Caldas, sur de Bolívar, Boyacá, Santander y Antioquia. Por lo tanto, nuestro reporte amplía el conocimiento de la distribución de esta especie a la región Orinoquía. La subespecie $H$. thoas nealces se registra para la vertiente oriental de la Cordillera Oriental, toda la costa pacífica, los valles de los ríos Cauca y Magdalena, la costa Caribe y el Catatumbo (ANDRADE, 2002; LECROM et al., 2002). Esta subespecie se encuentra registrada para el municipio de Arauca, por lo cual se 
amplía su rango de distribución a la región Orinoquía. De igual manera, registramos la presencia de la especie $H$. homothoas en la Orinoquia (municipios de Arauca y Paz de Ariporo), la cual previamente estaba documentada para el flanco oriental de la Cordillera Occidental y el flanco oriental de la Cordillera Oriental (ANDRADE, 2002; LECROM et al., 2002).

Por otro lado, se destaca que la subespecie $P$. eurimedes mycale no se encuentra reportada para Colombia de acuerdo al listado y distribución de especies de mariposas de Colombia (ANDRADE, 2002) y que de acuerdo a LECROM et al. (2002) y LAMAS (2004) esta subespecie se encuentra distribuida desde Panamá hasta el norte de Colombia. Mediante este trabajo se amplía su posible distribución a la región de la Orinoquia colombiana y el flanco oriental de la Cordillera Oriental. En el caso de B. polydamas polydamas, B. ingenuus, $H$. anchisiades idaeus, $P$. sesostris tarquinus y $P$. eurimedes eurimedes, el reporte concuerda con la distribución propuesta por ANDRADE (2002) en la región Orinoquia. En resumen, este trabajo amplía el conocimiento de la distribución geográfica de cuatro subespecies en la Orinoquia colombiana, ampliando el conocimiento a nivel regional.

Tabla 2. Comparación de la riqueza de especies reportadas para los departamentos de Meta, Casanare y Arauca. (La presencia se indica con una X).

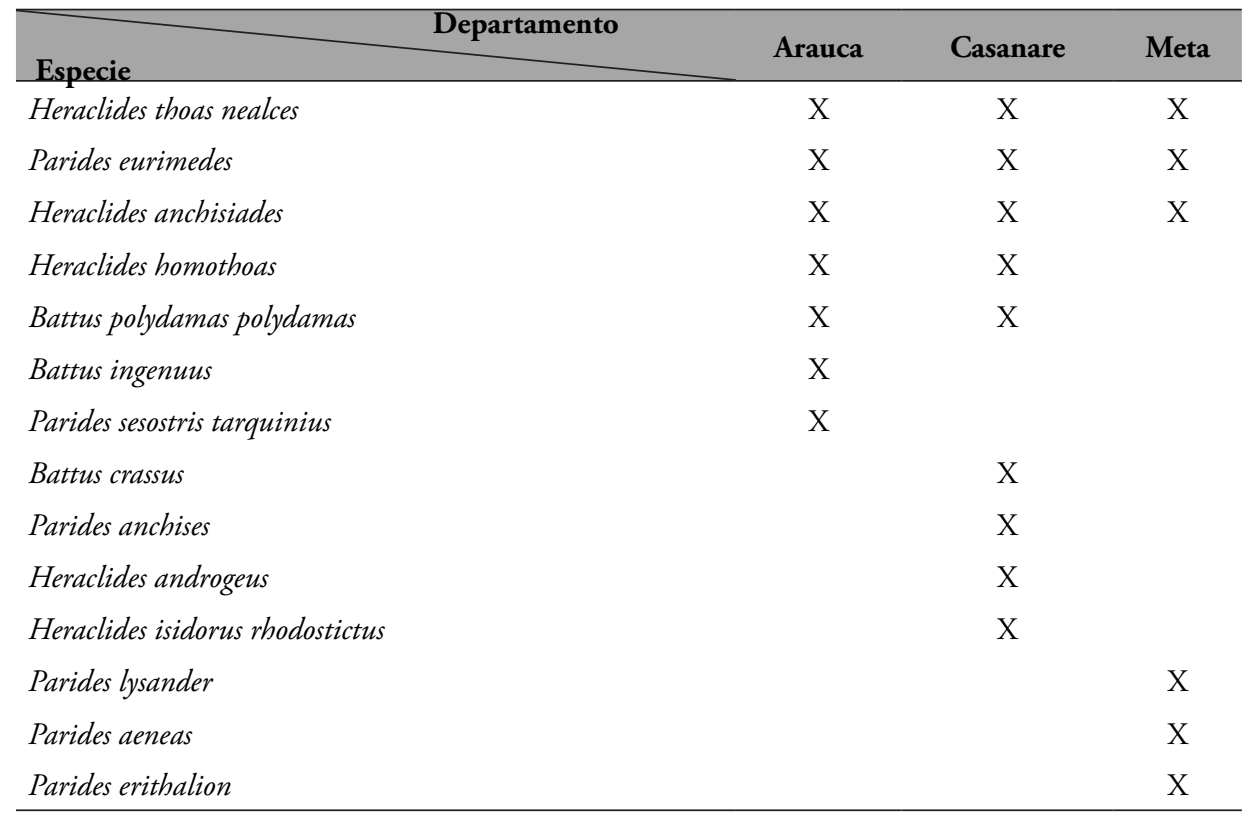

\section{AGRADECIMIENTOS}

Los autores de este trabajo quieren expresar su agradecimiento al profesor Gonzalo Andrade del Instituto de Ciencias Naturales (ICN) de la Universidad Nacional, por su ayuda en la corroboración e identificación de algunos de los especímenes citados en esta publicación. Al profesor Rodrigo Enrique Cárdenas Acevedo, Director de 
la sede Orinoquia de la Universidad Nacional de Colombia, por el apoyo irrestricto brindado a las colecciones biológicas de la sede desde su inicio. A los profesionales Yessenia Sanabria, Richard Jiménez y Francisco Mijares. Agradecemos además a los alumnos Ángel Matiz, Yilber Mina, Laura Mojica, Evelyn Gómez, Elizabeth Cuevas y Franklin Morales, quienes trabajaron de la mano con los autores en la curaduría y colecta de buena parte de los especímenes relacionados en este artículo.

\section{BIBLIOGRAFÍA}

ANDRADE, G., 2002.- Biodiversidad de las mariposas (Lepidóptera: Rhopalocera) de Colombia. 2: 153-172 (en) COSTA, C., VANIN, S.A., LOBO, M. \& MELIC, A. (eds.) Proyecto de Red Iberoamericana de Biogeografía y Entomología Sistemática. Lepidóptera tercer milenio. Sociedad Entomología Aragonesa, España.

APOLINAR, M., 1939.- Catálogo explicativo de las ropalóceras colombianas del Museo del Instituto La Salle. Rev. Ac. Col. Cienc. Ex. Fis. \& Nat., 3 (9/10): 108-111, pls. 1-2.

1940.- Catálogo explicativo de las ropalóceras colombianas del Museo del Instituto La Salle. Rev. Ac. Col. Cienc. Ex. Fis. \& Nat., 3 (12): 406-413, pls. 1-2.

ARBELÁEZ-CORTÉS, E., 2013.- Knowledge of Colombian biodiversity: Published and indexed. Biodivers Conserv., 22: 2875-2906.

FERNÁNDEZ, A., GONTO, R., RIAL, B.A., ROSALES, J., SALAMANCA, B., CÓRDOBA, M. et al., 2010.- Flora y vegetación de la cuenca del rio Orinoco 124-195 (en) LASSO, C.A., USMA, J.S., TRUJILLO, F. \& RIAL, A. (eds.) Biodiversidad de la cuenca del Orinoco: bases científicas para la identificación de áreas prioritarias para la conservación y uso sostenible de la biodiversidad. Instituto de Investigación de Recursos Biológicos Alexander von Humboldt, WWF Colombia, Fundación Omacha, Fundación La Salle e Instituto de Estudios de la Orinoquia (Universidad Nacional de Colombia), Bogotá, D.C., Colombia.

FRAIJA, F.N. \& FAJARDO, M.G.E., 2006.- Caracterización de la fauna del orden Lepidoptera (Rhophalocera) en cinco localidades de los Llanos Orientales colombianos. Acta Biol. Colomb., 11 (1): 55-68.

GARCÍA-ROBLEDO, C., CONSTANTINO, L.M., HEREDIA, M.D. \& KATTAN, G., 2002.- Mariposas comunes de la cordillera central de Colombia. Wildlife Conservation Society.

GROPP, R.E., 2003.- Are university natural science collections going extinct? BioScience, 54 (1): 67-74.

LAMAS, G., 2004.- Atlas of Neotropical Lepidoptera. Checklist: Part 4A. Hesperioidea - Papilionoidea. Scient. Publ., Gainesville, Florida, USA.

LECROM J.F., CONSTANTINO, L.M. \& SALAZAR, J.A., 2002.- Mariposas de Colombia. Tomo I: Papilionidae. Carlec Ltda., Colombia.

LLORENTE, B.J. \& MARTÍNEZ, A.L., 1998.- conservacionista de las mariposas mexicanas Papilionidae (Lepidoptera, Papilionoidea): 149-178. (en) RAMAMOORTHY, T.P., BYE, R., LOT, A. \& FA, J. (eds.) Diversidad Biológica de México: orígenes y distribución. Instituto de Biología, Universidad Nacional Autónoma de México, México, D.F.

MEDINA, U.C.A., FERNÁNDEZ, F. \& ANDRADE-C., M.G., 2010.- Insectos: escarabajos coprófagos, hormigas y mariposas: 196-215 (en) LASSO, C.A., USMA, J.S., TRUJILLO, F. \& RIAL, A. (eds.) Biodiversidad de la cuenca del Orinoco: bases científicas para la identificación de áreas prioritarias para la conservación y uso sostenible de la biodiversidad. Instituto de Investigación de Recursos Biológicos Alexander von Humboldt, WWF Colombia, Fundación Omacha, Fundación La Salle e Instituto de Estudios de la Orinoquia (Universidad Nacional de Colombia), Bogotá, D.C., Colombia.

PEÑA-PIRAZAN, J.D., 2003.- Minería y medio ambiente en Colombia. Una síntesis histórica de la mala administración de nuestros recursos naturales mineros de la degradación ambiental y de la generación conflictos sociales, asociados a desastres naturales. Una mirada hacia el futuro económico y ambiental de este importante sector productivo: Tesis de Especialización, Universidad Sergio Arboleda, Bogotá.

PONDER, W.F., CARTER, G.A., FLEMONS, P. \& CHAPMAN, R.R., 2001.-Evaluation of museum collection data for use in biodiversity assessment. Conserv. Biol., 15: 648-657.

QUINTERO, S.I., OSORIO, D.P., CASTILLO, R.M. \& HIGUERA, D.M., 2007.- Insectos: 87-113 (en) VILLARREALLEAL, H. \& MALDONADO-OCAMPO, J. (eds.) Caracterización biológica del Parque Nacional Natural El Tuparro (Sector noreste), Vichada, Colombia. Instituto de Investigación de Recursos Biológicos Alexander von Humboldt, Bogotá D.C., Colombia.

RIPPSTEIN G., AMÉZQUITA A., ESCOBAR, G. \& GROILLER C., 2001.- Condiciones naturales de la sabana: 1-22 (en) RIPPSTEIN G., ESCOBAR G., MOTTA F. (eds.) Agroecología y Biodiversidad de las Sabanas en los Llanos Orientales de Colombia. Centro Internacional de Agricultura Internacional, Cali, Colombia.

ROSALES, J., SUÁREZ, C.F. \& LASSO, C.A., 2010.- Descripción del medio natural de la cuenca del Orinoco: 52-74 (en) LASSO, C.A., USMA, J.S., TRUJILLO, F. \& RIAL, A. (eds.) Biodiversidad de la cuenca del Orinoco: bases científicas para la identificación de áreas prioritarias para la conservación y uso sostenible de la biodiversidad. Instituto de Investigación de Recursos Biológicos Alexander von Humboldt, WWF Colombia, Fundación Omacha, Fundación La Salle e Instituto de Estudios de la Orinoquia (Universidad Nacional de Colombia), Bogotá, D.C., Colombia. 
ROTHSCHILD, W.L. \& JORDAN, K., 1906.- A revision of the American papilios. Novitates Zoologicae, 13 (3): 411-752, + IX plates. Facsimile. Ph. Arnaud, Jr. (ed.) [1967].

SUÁREZ, A.V. \& TSUTSUI, N.D., 2004.- The value of museum collections for research and society. BioScience, 54: 66-74.

VILLARREAL, H., ÁLVAREZ, M., CÓRDOBA, S., ESCOBAR, F., FAGUA, G., GAST, F. et al., 2006.- Programa de Inventarios de Biodiversidad. Segunda edición. Instituto de Investigación de Recursos Biológicos Alexander von Humboldt, Bogotá, Colombia.

YARA-ORTIZ, C.L., PEÑA, J.M. \& URBANO, T.P., 2011.- Hormigas y Mariposas del Casanare: 102-119 (en) USMA, J.S. \& TRUJILLO, F. (eds.) Biodiversidad del Casanare: Ecosistemas Estratégicos del Departamento. Gobernación de Casanare. WWF, Bogotá D.C., Colombia.

Anexo I. Registros del material revisado.

\section{TAXONOMÍA}

Superfamilia Papilionoidea (Latreille, 1802)

Familia Papilionidae (Latreille, 1802)

Subfamilia Papilioninae (Latreille, 1802)

Tribu Troidini (Talbot, 1939)

Género Battus (Scopoli, 1777)

Battus ingenuus (Dyar, 1907)

Material revisado:

COLOMBIA, Arauca, Tame, vereda Rincón Hondo. 212 msnm. 6²7’24,10”N 7140'19,42”W. 3-ago-2013. leg. Matiz A. ㅇ CEO-1788.

Battus polydamas polydamas (Linnaeus, 1758)

Material revisado:

COLOMBIA, Arauca, Arauca, casa de habitación, casco urbano. $133 \mathrm{msnm}$. 705’05,3300”'N 7045’25,3100”W. 29-sep-2010. leg. Gómez E. ○̊ CEO-44.

Arauca, Arauca, Campus UN, bosque secundario. $130 \mathrm{msnm} .7^{\circ} 00^{\prime 33}, 6358^{\prime \prime} \mathrm{N}$ 7044'33,0626”'W. 23-ago-2010. leg. Parra V.G. s.g. CEO-143.

Arauca, Arauca, finca Las Mercedes, rastrojo. 126 msnm. 6 57’30,3778'N 7042'54,2601'”W. 11-feb-2012. leg. Pérez B.N. ô CEO-144.

Arauca, Arauca, vereda Chaparrito, almorzadero. 130 msnm. 659'51,1887'N 7046'17,9044’’W. 9-nov-2011. leg. Pérez B.N. §̊ CEO-145.

Aruca, Arauca, finca Las Mercedes, rastrojo. 126 msnm. 657’30,3778’N 7042'54,2601’'W. 11-feb-2011. leg. Pérez B.N. § CEO-146.

Arauca, Arauca, Campus UN, zonas verdes. 130 msnm. 700'46,5925'N 7044'41,4189”'W. 1-jun-2011. leg. Pérez B.N. ô CEO-150.

Arauca, Arauca, Campus UN, ecotono entre sabana inundable, pastizal y borde de matas de monte. 128 msnm. 700'30,4000”N 7044'32,8000”W. 19-nov-2012. leg. 
Sanabria Y. ô CEO-1068.

Arauca, Arauca, vereda El Rosario, caño El Papayito vía Arauca-Tame. 130 msnm. 654'29,47’'N 7053'5,72’'W. 26-jun-2013. leg. Pérez B.N. §̊ CEO-1657.

Arauca, Tame, vereda Santa Inés. 261 msnm. 6³0’40,00”N 7143’28,05’W. 13-ene2013. leg. Mina Y. o CEO-1782.

Arauca, Arauca, Campus UN sede Orinoquia, corredor. 130 msnm. $7^{\circ} 00^{\prime} 47,13$ ”N 7044’40,65”W. 30-jun-2013. leg. Pérez B.N. ô CEO-1784.

Arauca, Arauca, vereda San Ramón, finca Rancho Bonito. 120 msnm. 644’53,25”N 70³8’54,53’'W. 10-jun-2013. leg. Jiménez R.Y. § CEO-1785.

Arauca, Arauca, vereda San Ramón, finca Rancho Bonito. 120 msnm. 644’53,25”N 70³8'54,53’'W. 10-jun-2013. leg. Jiménez R.Y. + CEO-1786.

Arauca, Arauca, vereda San Ramón, finca Rancho Bonito. 124 msnm. 644’56,74”N 70³8'51,68’W. 10-jun-2013. leg. Jiménez R.Y. + CEO-2207.

Casanare, Paz de Ariporo, vereda Montañas del Totumo. 153 msnm. 555"39,33"'N 71¹4'7,89'”'W. 7-jul-2013. Leg. Matiz A. o CEO-1783.

Casanare, Paz de Ariporo, vereda Montañas del Totumo. 153 msnm. 555"39,33"'N $71^{\circ} 14^{\prime} 7,89^{\prime \prime \prime}$ W. 5-jul-2013. leg. Matiz A. ㅇ CEO-1787.

Género Parides (Hübner, 1819)

Parides eurimedes (Stoll, 1782)

Parides eurimedes arriphus (Boisduval, 1836)

Material revisado:

COLOMBIA, Arauca, Arauca, Campus UN, zonas verdes. 129 msnm 700’47,5900’'N 7044’40,5500’W 23-ago-2012. leg. Pérez B.N. §̋ CEO-646.

Arauca, Arauca, Campus UN, entre sabana inundable y matas de monte. $128 \mathrm{msnm}$. 700’32,2000”'N 7044’33,5200”'W. 3-nov-2012. leg. Sanabria Y. ô CEO-1146.

Arauca, Tame, zona urbana, Calle 8 \#8-39 barrio Sucre. 315 msnm. 6 27’30,35’N 7143’22,35’'W. 16-jun-2013. leg. Matiz A. + CEO-1654.

Arauca, Tame, vereda Santa Inés. 261 msnm. 6³0'40,00”'N 7143'28,05'”. 2-dic2012. leg. Mina Y. ô CEO-1780.

Arauca, Tame, vereda Santa Inés. 261 msnm. 6³0’40,00”N 7143’28,05”W. 10-ene2013. leg. Mina Y. ô CEO-1781.

Arauca, Tame, vereda Rincón Hondo. 212 msnm. 6²7’24,10”N 7140'19,42'”W. 3-ago-2013. leg. Matiz A. đै CEO-1789.

Arauca, Tame, vereda Mapoy. 200 msnm. 6¹6’52,37’N 7142’3,86”'W. 15-jul-2013. 
leg. Matiz A. + CEO-1790.

Arauca, Arauca, vereda Mata de gallina, finca Los Laureles. 128 msnm. 659'29,19'N 7047’52,98’'W. 14-dic-2013. leg. Agudelo J. o CEO-2242.

Arauca, Saravena, vereda El Pescado, finca Alicia. 214 msnm. 659'52,82'N 7154’08,81’W. 11-nov-2013. leg. Pérez N.B. ㅇ CEO-1969.

Arauca, Arauca, Campus UN, biblioteca. 130 msnm. $7^{\circ} 00^{\prime} 46,1418^{\prime \prime} \mathrm{N}$ 7044’40,0815”'W. 18-nov-2013. leg. Pérez N.B. + CEO-1968.

Arauca, Arauca, Campus UN, biblioteca. 130 msnm. $7^{\circ} 00^{\prime} 46,1418^{\prime \prime} \mathrm{N}$ 7044’40,0815’'W. 18-nov-2013. leg. Pérez N.B. § CEO-1967.

Arauca, Tame, vereda El Susto. 195 msnm. 6¹4'54,18”N 7141'19,86”'W. 28-dic2013. leg. Matiz A. + CEO-2302.

Arauca, Tame, vereda El Susto. 195 msnm. 6¹4'54,18”N 7141'19,86”W. 28-dic2013. leg. Matiz A. ㅇ CEO-2300.

Arauca, Tame, vereda Cravo Totumo. 221 msnm. 6³1'3,66”'N 71'39'55,50'W. 29dic-2013. leg. Matiz A. o CEO-2302.

Arauca, Arauca, Campus UN, biblioteca. 128 msnm. 700'46,1418'N 7044'40,0815”'W. 28-sep-2011. leg. Pérez B.N. ㅇ CEO-52.

Arauca, Arauca, Campus UN. 128 msnm. 700’48,8100”'N 7044’42,4200”'W. 2-jul2010. leg. Goméz E. o CEO-105.

Arauca, Arauca, campus UN, entre sabana inundable, borde de terraplén y cobertura arbórea Trampa \# 1, zona 2. 130 msnm. 700'44,6104'” 7044'40,4667'”W. 14-sep-2012. leg. Sanabria Y. q CEO-720.

Arauca, Tame, pastizal - zona urbana. 274 msnm. 6²7’30,6600”'N 7143'19,0100”'W. 5-nov-2012. leg. Pérez B.N. o CEO-1147.

Arauca, Tame, vereda Cravo Totumo. 220 msnm. 6³2'20,17'N 7140'53,75'”. 15-jun-2013. leg. Matiz A. + CEO-1653.

Arauca, Saravena, vereda El Pescado, finca Alicia. 214 msnm. 65952,82’N 715408,81’W. 11-nov-2013. leg. Pérez N.B. + CEO-2037.

Arauca, Saravena, vereda El Pescado, finca Alicia. 214 msnm. 659'52,82'N 7154’08,81’'W. 11-nov-2013. leg. Pérez N.B. ㅇ CEO-1971.

\section{Parides eurimedes eurimedes (Stoll, 1782)}

Material revisado:

COLOMBIA, Arauca, Tame, bosque de galería. 311 msnm. 6²8'02,3977’N 7144'31,6887’'W. 13-jul-2011. leg. Pérez B.N. q CEO-51. 
Parides eurimedes mycale (Godman \& Salvin, 1890)

Material revisado:

COLOMBIA, Arauca, Campus UN, biblioteca. 130 msnm. $7^{\circ} 00^{\prime} 46,1418^{\prime \prime} \mathrm{N}$ 7044'40,0815’'W. 18-nov-2013. leg. Pérez N.B. + CEO-1970.

Parides sesostris (Cramer, 1779)

Parides sesostris tarquinus (Boisduval, 1836)

Material revisado:

COLOMBIA, Arauca, Arauquita, corregimiento La Esmeralda, casa de habitación.

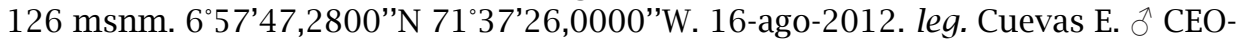
640.

Arauca, Tame, vereda Rincón Hondo. 212 msnm. 6²7’24,10”N 7140'19,42”'W. 3-jul-2013. leg. Matiz A. q CEO-1791.

Tribu Papilionini (Latreille, 1802)

Género Heraclides (Hübner, 1819)

Heraclides anchisiades (Esper, 1788)

Heraclides anchisiades idaeus (Fabricius, 1793)

Material revisado:

COLOMBIA, Arauca, Arauca, Campus UN. 128 msnm. 700'48,8100'N 7044’42,4200’'W 13-sep-2012. leg. Morales G F. §̊ CEO-723.

Arauca, Arauca, Campus UN Orinoquia, biblioteca. 128 msnm. 700'46,1418”N 7044'40,0815'W. 23-nov-2013. leg. Mijares F. ô CEO-1950.

Arauca, Arauca, Campus UN Orinoquia, biblioteca. 128 msnm. 700'46,1418’N 7044'40,0815’'W. 18-sep-2013. leg. Pérez N.B. † CEO-2187.

\section{Heraclides homothoas (Rothschild \& Jordan, 1906)}

Material revisado:

COLOMBIA, Arauca, Arauca, vereda Chaparrito, almorzadero, borde carretera. 130 msnm. 659'51,1887’'N 7046’17,9058’'W. 9-nov-2011. leg. Pérez B.N. + CEO-45.

Arauca, Arauca, casa de habitación. 133 msnm. 705’05,3300”N 7045'25,3100”'W. 30-sep-2010. leg. Gómez E. + CEO-151.

Arauca, Arauca, vereda El Rosario, caño El Papayito vía Arauca-Tame. 130 msnm. 654'29,47’N 7053'5,72”'W. 26-may-2013. leg. Pérez B.N. đo CEO-1659.

Arauca, Arauca, vereda San Ramón, finca Rancho Bonito. 120 msnm. 644'53,25”N 70³8'54,53”'W. 10-jun-2013. leg. Jiménez R.Y. s.g. CEO-1793. 
Arauca, Arauca, vereda Mata de Gallina, finca Los Laureles. 128 msnm. 659'29,19’'N 7047’52,98”'W. 1-dic-2013. leg. Pérez N.B. + CEO-2087;

Casanare, Paz de Ariporo, vereda Montañas del Totumo. 153 msnm. 55539,33”N 71'14'7,89’'W. 7-jul-2013. leg. Matiz A. q CEO-1792.

Heraclides thoas (Linnaeus, 1771)

Heraclides thoas nealces (Rothschild \& Jordan, 1906)

Material revisado:

COLOMBIA, Arauca, Fortul, camino secundario vehicular. 229 msnm. 647’37,2115’'N 7145’55,1266”'W. 25-jun-2011. leg. Pérez B.N. ô CEO-46.

Arauca, Tame, zona urbana, Calle 8 \#8-39 barrio Sucre. 315 msnm. 6 27"0,35”N 7143’22,35’'W. 16-jun-2013. leg. Matíz A. + CEO-1655;.

Arauca, Arauca, vereda El Rosario, caño El Papayito vía Arauca-Tame. 130 msnm. 654'29,47’N 7053’5,72’'W. 26-may-2013. leg. Pérez B.N. ô CEO-1660.

Arauca, Tame, vereda Santa Inés. 261 msnm. 6³0’40,00”'N 7143'28,05'O. 9-dic2012. leg. Mina Y. o CEO-1778.

Arauca, Tame, vereda Santa Inés. 261 msnm. 6³0’40,00”N 7143’28,05”'W. 11-ene2013. leg. Mina Y. o CEO-1779.

Norte de Santander, Toledo, quebrada La China. 494 msnm. 702'20,7'N 72¹3’09”'W. 3-may-2013. leg. Jiménez R. §̋ CEO-1640. 